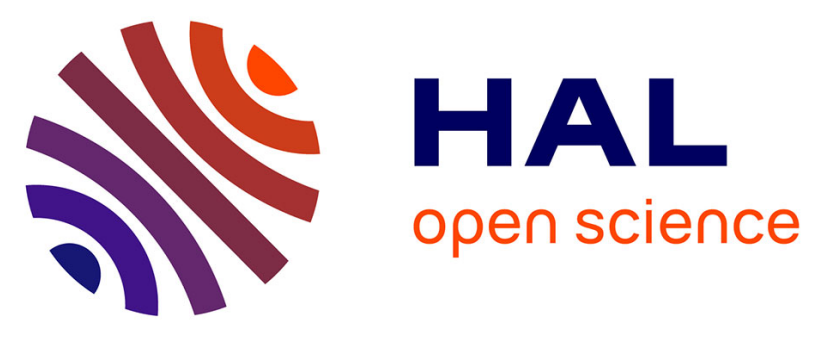

\title{
Investigation on the capabilities of low-cost and smartphone-based coastal imagery for deriving coastal state video indicators: applications on the upper mediterranean
}

Nico Valentini, Yann Balouin, Thibault Laigre, Clement Bouvier, Rémi Belon, Alessandra Saponieri

\section{To cite this version:}

Nico Valentini, Yann Balouin, Thibault Laigre, Clement Bouvier, Rémi Belon, et al.. Investigation on the capabilities of low-cost and smartphone-based coastal imagery for deriving coastal state video indicators: applications on the upper mediterranean. Coastal Sediments 2019, May 2019, Tampa, United States. , 2019. hal-02076694

\section{HAL Id: hal-02076694 https: / hal-brgm.archives-ouvertes.fr/hal-02076694}

Submitted on 22 Mar 2019

HAL is a multi-disciplinary open access archive for the deposit and dissemination of scientific research documents, whether they are published or not. The documents may come from teaching and research institutions in France or abroad, or from public or private research centers.
L'archive ouverte pluridisciplinaire HAL, est destinée au dépôt et à la diffusion de documents scientifiques de niveau recherche, publiés ou non, émanant des établissements d'enseignement et de recherche français ou étrangers, des laboratoires publics ou privés. 


\title{
INVESTIGATION ON THE CAPABILITIES OF LOW-COST AND SMARTPHONE-BASED COASTAL IMAGERY FOR DERIVING COASTAL STATE VIDEO INDICATORS: APPLICATIONS ON THE UPPER MEDITERRANEAN
}

\author{
NICO VALENTINI ${ }^{1}$, YANN BALOUIN ${ }^{1}$, THIBAULT LAIGRE $^{1}$, CLEMENT $^{3}$ \\ BOUVIER $^{1.2}$, REMI BELON $^{1}$, ALESSANDRA SAPONIERI ${ }^{3}$
}

1. BRGM, Univ. Montpellier, 1039, rue de Pinville, 34000 Montpellier, France. n.valentini@brgm.fr; y.balouin@brgm.fr; c.bouvier@brgm.fr; r.belon@brgm.fr.

2. CNRS, UMR EPOC, Univ. Bordeaux, Allée Geoffroy Saint-Hilaire, CS 50023, 33615 Pessac Cedex, France.clement.bouvier@u-bordeaux.fr.

3. Department of Civil, Environmental, Building Engineering and Chemistry (DICATECh), Politecnico di Bari, Bari 70126, Italy. alessandra.saponieri@poliba.it.

\begin{abstract}
This work deals with the implementation and operational use of lowcost and smartphone-based camera system for the purpose of coastal video monitoring. Capability of such system for coastal remote sensing is described in this study. Particularly, a small network of (CSolarcam has been implemented in Corsica Island (France) for purposes of coastline evolution, sandbar(s) detections and to investigate the influence of the Posidonia banquettes on morphological evolution. A new coastal indicator derived from video observation is presented. Furthermore, the second part deals with the description and application of image semantic segmentation procedures, developed in order to regionalize the main morphologies framed by web-cams. Such techniques can be apply for a range of applications: from shoreline evolution, sandbar(s) detection, algae identification and development of a warning system.
\end{abstract}

\section{Introduction}

Nowadays, worldwide coastal zone is heavily exposed to several hazards, related to wide urbanization and human activities, as well as climate changes effects, which tend to weaken its environmental quality and sustainability. Coastal management retains a special role to shape economic and social resources, particularly for places with huge sea resources. To accomplish such a worthy scope, coastal monitoring measurements have been established to represent a leading phase to collect meaningful data, useful for studies related to hydrodynamic processes and morphological evolution. Specifically, remotesensed imagery is considered as a very helpful tool for coastal monitoring field. Since the first experiences, during the 1980s, it has been applied for a variety of objectives, aiming at studying nearshore hydrodynamics and morphodynamics (e.g. shoreline and nearshore bars detection, beach width, breaker zone and intertidal bathymetry estimations, runup or swash investigations). Currently, the 
'state of the art' in coastal imaging is represented by video systems, among which the most known are Argus (Holman and Stanley 2007) and VM4GIS (Rihouey et al. 2009) that typically include more fixed cameras per site, on-site acquisition, control and different suites for data analysis. They are installed in case of necessity and their dissemination could be actually hampered by economic constraints (Valentini, Damiani, Molfetta, \& Saponier, 2016).

Principal objective of the present contribution is to discuss about the capabilities of low elevation, low-cost smartphone imagery (CSolarcam) in the investigation of coastal processes for obtaining qualitative and quantitative coastal state indicators, by applying also new semantic segmentation techniques. Such experimentations in the Mediterranean area will be described and complemented with the development and implementation of a warning system for Sargassum macroalgae in the Caribbean.

\section{Study site and system description}

The (C) Solarcam network developed in Corsica Island (France) consists of three pilot sites: Ostriconi, Meria and Pietracorbara (see fig. 1). They are pocket beaches, chosen also because of the subsistence of Posidonia Oceanica meadows, characterized by accessibility to good view points for webcam installation, and by different wave exposures (Balouin, Bélon, Merour, \& Riotte, 2014). The CSolarcam system (https://www.solarcam.fr) is composed of a standalone camera powered by a modified Sony Android smartphone that provides timelapse in real time, a small solar panel (for charging), and a battery that keeps the system operating even during bad weather conditions. Up to one image every minute is transmitted over $3 \mathrm{G}$, but the interval is adjustable, depending obviously on the solar panel efficiency and mainly on the scopes. Images, with 8 Megapixels resolution, are stored directly to a FTP server. A plastic case for hosting the phone and a plastic band for attaching it to a support (e.g. pole, tree, etc.) are used. The price of the described system (expect the $3 \mathrm{G}$ monthly communication expenses) is less than $370 €$. It guarantees good portability, cheapness, simplicity in communication and installation, good resolution; meanwhile issues are mainly related to network signal fluctuations, low acquisition frequency and potential movements due to strong winds or temperature-related material deformations of case/support. In order to investigate morphological and hydrodynamic coastal processes, particularly coastline evolution, Posidonia banquettes and their influence on coastline behavior, bar dynamics, etc., modelling chains have been developed. A Matlabbased GUI has been built aiming images processing and chain modelling. The implemented processing tools include averaging, geo-rectification, sandbar(s)/shoreline or algae detection with semi-automatic analysis and automatic semantic segmentation. The wave data derived from the Wave 
Forecast AEMET/OPPE organism, which distribute hourly wave and wind fields forecast for the Northern Atlantic and the Western Mediterranean area (http://www.puertos.es/en-us).

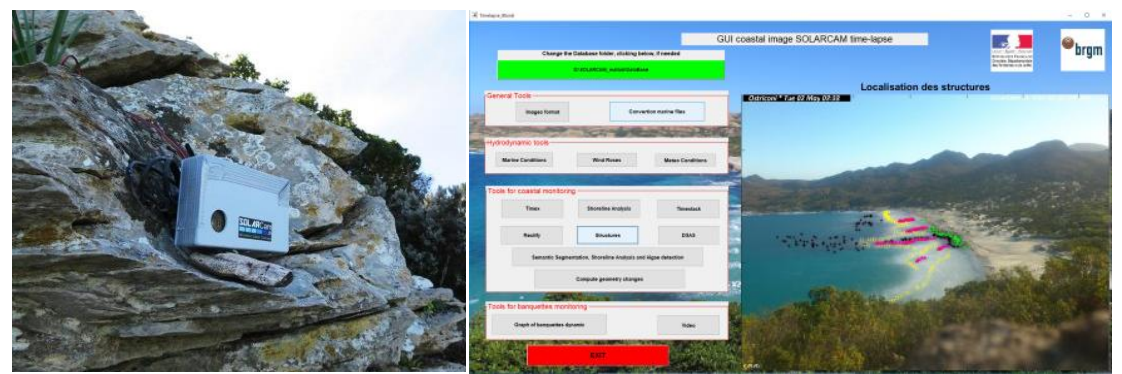

Fig. 1. (a) Example of solarcam placement for the monitoring of Ostriconi's beach. (b) Solarcam GUI main interface and an exemplary image of localized emerged and submerged structures, from nearshore bars to algae.

\section{Posidonia dynamics - results}

The system described above has been applied, among other scopes, for analyzed the dynamics of the Posidonia' banquettes. A general dynamics has been identified at these pocket beaches, characterized by two general mechanisms of deposit: lateral deposition and subsequent accumulation (Laigre, 2017). The former is mainly due to low to moderate wave climate, the latter during rough wave conditions. A thresholds criterion has been clearly discernable: low wave conditions (under a low threshold) favor the banquettes to remain stable with a tendency to sink, while over a high threshold, common destruction of deposits have been observed. This kind of threshold criterion is highly influenced by the compactness of the banquettes: the greater the compactness, the rougher the wave conditions useful for allowing the deposit displacements. The main banquettes motions have been identified in the range between these mentioned thresholds, which evidently are different among beaches, influenced by the foreshore and nearshore morphology. Basically, the banquettes behavior followed beach morphological behavior like beach rotation, but could also influence hydrodynamics and sediment transport. The wave direction is obviously responsible for the construction and destruction locations of the zone involved. 
As an example, two representative hydrodynamic events at the Ostriconi's beach are described in the following, the former from $01^{\text {st }}$ to $03^{\text {rd }}$, the latter from $09^{\text {th }}$ to $11^{\text {th }}$ April of 2017 . The two successive events are featured by North and South wave direction with a duration of almost two days. Fig. 2 shows the rectified time-exposure images representative of both events, with initial
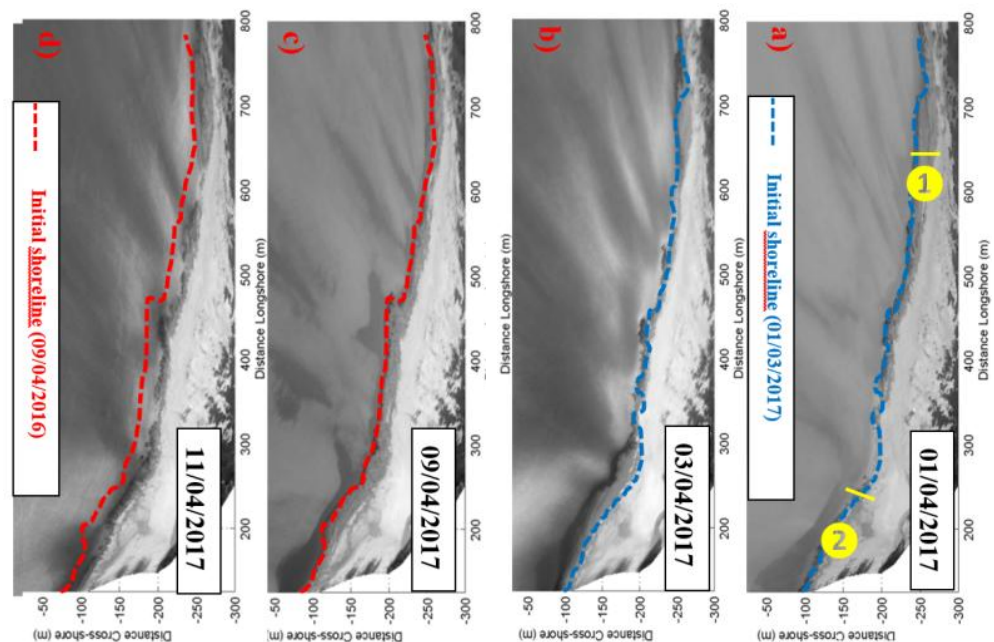

Fig. 2. Rectified images of (a) 01/04, (b) 03/04, (c) 09/04 and of (d) 11/04/2017 with overlapped corresponding shorelines (blue and red). In yellow two cross-shore timestacks monitored over time at the monitored station.

shoreline position (blue and red dashed lines, respectively). Shoreline evolution, focusing on erosion and accretion phenomena is illustrated Fig. 3a for several cross-shore transects. During the first event, a global transfer of algae has been derived, with the formation of big deposit at the south side. Conversely, the second event produced a successive migration of Posidonia towards the northern side of the pocket beach. 
The image analysis are traditionally very useful in order to determine the 
position and evolution of submerged bars and rip channels (Balouin, 
Longueville, \& Colombet, 2016). In this study the distribution of leaves in water 


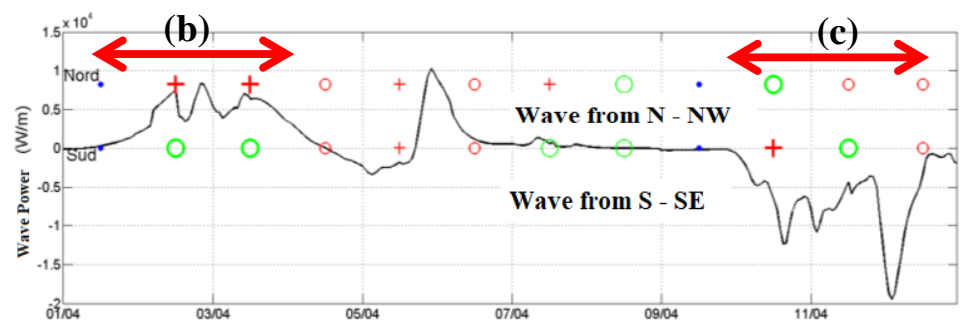

(a)

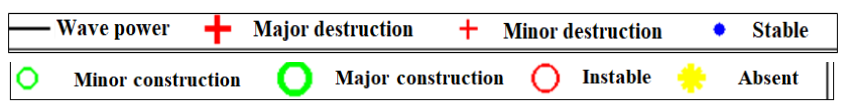

(b)
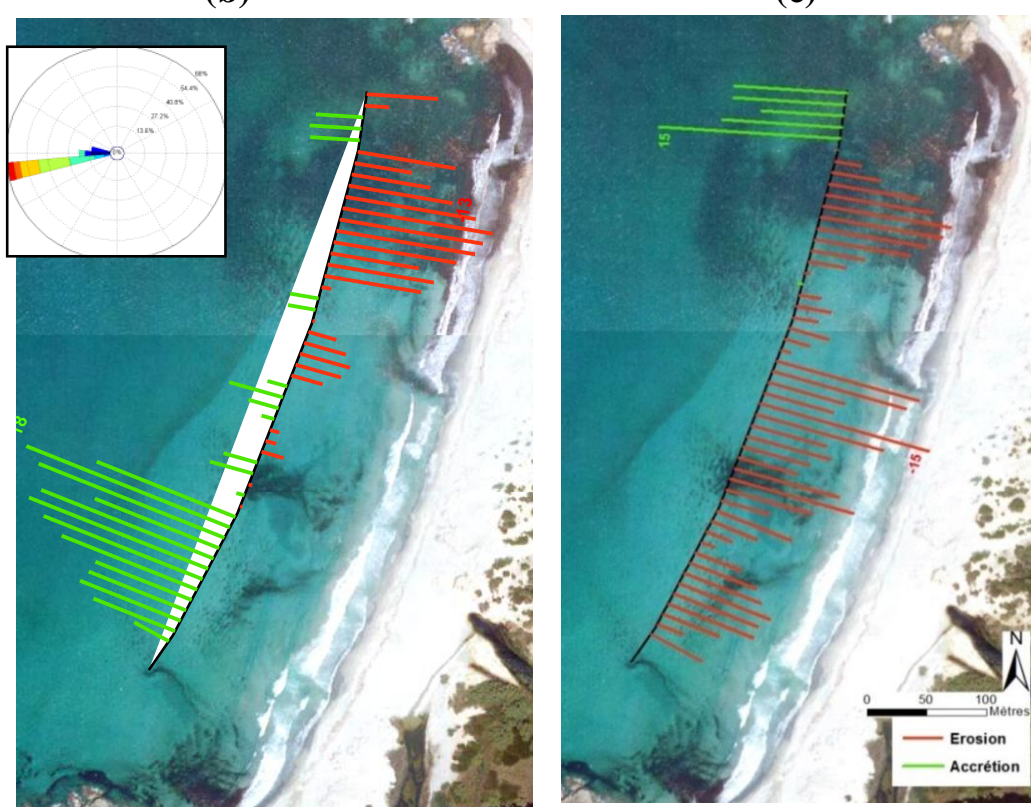

Fig. 3. Wave power calculated from offshore wave forecast data, with qualitative state indicator on the banquettes behaviors, derived from processing image with semi-automatic routines. Shoreline evolution in terms of erosion and accretion transects, computed for both wave events, wave rose overlapped.

were highly related with nearshore morphological pattern. Offshore, leaves are mainly sparse but while approaching the shore the leaves tended to be funneled in the rip channel, and then followed the two main deposit mechanisms, described above. Submerged sandbars morphology (i.e. see Fig. 2c where white dissipation patterns are visible) in reality have been shown to determine a strong 
influence on both direction of the leaves movements. Hence, the nearshore bar/rip current system represents an important factor influencing the development and distribution of the banquettes.

\section{Semantic image segmentation}

Image segmentation task aims to assign each pixel of an image a visual object class label from a selected range of possible classes. Semantic segmentation could be described as the process of associating each pixel of an image with a class label (i.e. coastal morphologies: sand, dune, vegetation, foam, surf, sky, sea, etc.). The exploitation of deep learning, a subset of machine learning, for semantic classification of imagery, does not directly require features to be detected. They are automatically learned from data, by using general-purpose procedures. A powerful and popular class of these algorithms can be defined as the deep convolutional neural networks $(D C N N)$, often trained on generic libraries, with attention on natural environment, road or indoor scenes $(\mathrm{He}$, Zhang, Ren, \& Sun, 2015; Krizhevsky, Sutskever, \& Hinton, 2012). The possibility to exploit the efficacy of deep neural networks trained for such generic purposes resides on procedures of transfer learning (Buscombe and Ritchie 2018). MobileNetV2 (Sandler, Howard, Zhu, Zhmoginov, \& Chen, 2018), among others, is a small and very efficient neural network, faster to train and execute, compared to competing architectures, designed to be transferable for generic image recognition tasks. By combining deep learning models which significantly outperforms earlier methods in many image processing tasks with traditional fully connected conditional random field $(C R F)$ could even achieve better results. The drawbacks of convolutional networks related to low spacial accuracy could be mitigated by dense CRF algorithm, which takes in account the low-level information captured by the local interactions of pixels and edges (Chen, Papandreou, Kokkinos, Murphy, \& Yuille, 2014; Liu \& Wen, n.d.).

Moreover, one can say the interest in coastal image analysis is highly related to the identification of semantic boundaries, which requires an association of precise boundaries to a semantic class, associated to them. This idea is typically seen as a combination of boundary detection and semantic segmentation (Hariharan, Arbelaez, Bourdev, Maji, \& Malik, 2011; Maninis, Pont-Tuset, Arbeláez, \& Van Gool, 2017). A common approach to this task is to separate method such as semantic segmentation and contour detection, and hence fuse the results of both steps. An alternative, simpler and straightforward way to improve the semantic segmentation output could be based on the inclusion of the effects of boundary detection by employing an edge-adhesive superpixels map. 
A modelling chain aiming the semantic segmentation of the coastal image has been developed which takes advantage of the above-mentioned processes. It consists of a training procedure of convolutional neural network and the implementation of semantic segmentation routine for pixel-wise classification of oblique images. Firstly, a workflow modified from Buscombe and Ritchie, 2018 for efficiently creating labeled imagery is employed. It is composed of a routine for interactively manual delineation of few regions in the input image defining specific classes in conjunction with CRF to estimate a pixel-scale segmentation, by using the inference algorithm for fully connected CRF models in which the pairwise edge potentials are defined by a linear combination of Gaussian kernels (Abadi et al., 2016). Sticky edge-adhesive superpixels are generated and snapped to the semantic class map to refine results. The superpixels are computed on the base of the original preprocessed (contrast-enhanced, shapepreserving be-exponential filtered) image by sticking the SEEDS superpixels, based on Bergh et al., 2013, to the structured edges computed with Dollar and Zitnick, 2015. The superpixels stack to the edges result in higher quality boundaries. The segmentation classes from CRF are snapped to the computed superpixels by majority voting of the regions: in particular, the superpixels that overlap more than $60 \%$ with the semantic class are assigned the corresponding label. Then, the re-training process of DCNN MobileNetV2 model is performed by using image patches of 96 x 96 pixels. This process has been carried out in Python environment with mainly TensorFlow and Tensorflow Hub (Abadi et al, 2016).

The routine aiming the pixel-wide classification substantially makes use of the re-trained DCNN model (MobilenetV2 network) for classes' prediction on image patches from unseen images. Then, the probabilistic approach, based on CRF above described, is applied in order to detail the information at the scale of pixel. Finally, the sticky edge-adhesive superpixels, computed with the method above described, allows further refining the final segmentation map. The parameters optimization of the processes are here not fully discussed. This generic procedure could be adopted for several type of monitoring activities, employing any kind of image sources. It turns out that by using high-resolution images (i.e. 8 Mega pix) a labelled database of around 12/15 images could generate a good training database of thousands of patches, which could be sufficient for reaching over $90 \%$ of validation precision.

\section{Shoreline analysis}

The procedure described in $\underline{\text { Sec. } 3}$ has been implemented for different beach morphologies. A quantitative assessment of the results is proposed in terms of shoreline detection capabilities apply for the Ostriconi's coastal stretch. 
As an example, the predicted classes overlay on the original oblique timex image (averaged over two hours), are shown in Fig. 4a. For this application the DCNN model has been trained with an averaged value of 4000 image patches

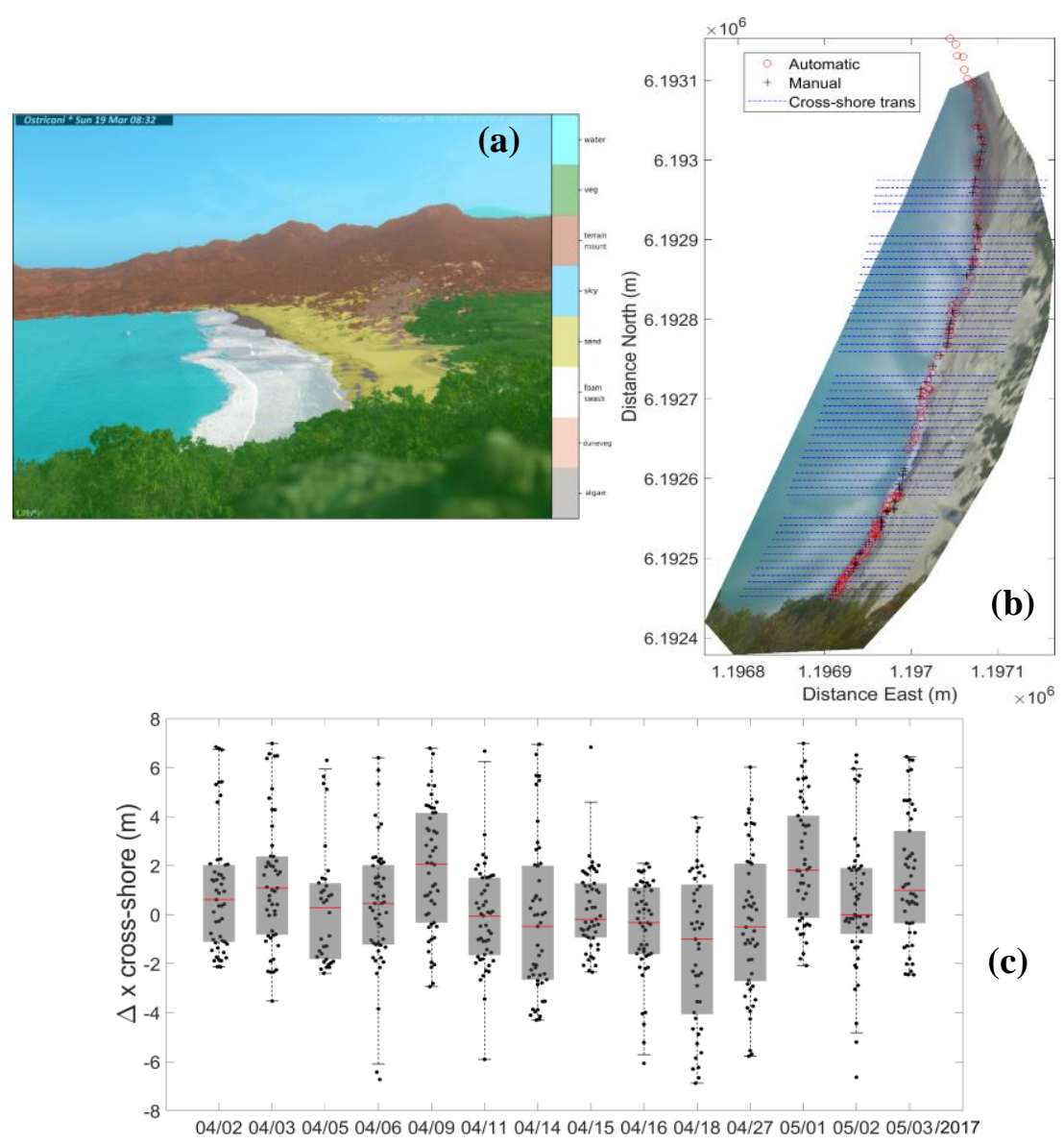

Fig. 4. (a). Example of semantic segmentation of timex oblique image at Ostriconi. (b). Overlay of manual, automatic shoreline, cross-shore transects on georectified timex image. (c). Statistic box plots of differences between manual and automatic results.

for each class (see colormap and legend for classes reference on Fig. 4a), by using 15 images. The validation accuracy obtained has been calculated as 0.915 , while the training accuracy as 0.932 , by using 1000 training steps and 0.01 as learning rate. Most training performance variation can be normally attributed to few hyperparameters of neural network (default network values are here applied) which, given the further detailing and refining processes here applied, for this application have not been optimized. 
In order to derive the shoreline from long-exposure images, the sea/sand interface is typically used as a proxy. Specifically, considered the several classes used in this context, the shoreline as boundary between the two blocks of algaesand classes and foam-sea classes has been chosen. We selected to assess the quality of results by employing the relative distances between the manual and automatic shorelines $(\Delta x)$ in real world coordinates, here presented for 14 datetimes (images). Both, the main errors derived from the quality of segmentation and detection themselves and the one due to georectification procedure are condensed in the statistics. The distribution of errors are displayed as box plot in Fig. 4c, for each analyzed image, during the time interval ranging between the $4^{\text {th }}$ of April and the $5^{\text {th }}$ of May 2017 (not all the days are included). For the most of images, the sample dispersion which is represented by the distance between the upper and lower quartiles, is close to the median, suggesting that over $50 \%$ of data is concentrated around the median value. The overall averaged value calculated is less than $1.8 \mathrm{~m}$ with a root mean square error of around $2.5 \mathrm{~m}$. Outliers of the box plot can be associated to further transects, where the image footprints equals the value of around $10 \mathrm{~m}$. They are also associated with the beached Posidonia banquettes, with a swash interface difficult to well localize. Generally, the automatic results seems promising in terms of relative distances to manual drawings.

\section{Sargassum warning system}

Recently, there's a growning need for the quantification of the spatio-temporal pattern of floating/beached macroalgae, particularly Sargassum species and remote/ground sensing application represents an interesting tool. Sargassum is particularly distributed along the Gulf of Mexico and the Caribbean, representing important ecological and economic interests. However, an excessive growth has generated massive off-shore Sargassum shoals that negatively affect coastal towns and certain coastal ecosystems (Cuevas, UribeMartínez, \& Liceaga-Correa, 2018). It has a negative effect on the tourism on which much of the region depends - the sight and smell left beaches highly unattractive, and swimming appears impossible. The issue is being taken so seriously from governments across the Caribbean that they have initiated emergency programmes to deal with this big issue.

In the effort of extending the capability of the CSolarcam video system and the automatic semantic segmentation, described in Sec. 3, a modelling chain is being exploited aiming the development of a warning system to assist the local government and municipalities in quantifying the beached/floating macroalgae. This quantification plays a crucial role for defining strategies for collection (i.e. trucks number and capacity) rather than the storage, and eventually the recycling and/or reuse activities. 
The processing chain makes use of a three-step procedure: (i) collecting necessary information of image and performing an automatic segmentation of the coastal stretch investigated. (ii) Analyzing the predicted classes in order to detect the potential Sargassum bloom/banquettes blobs, filtering based on relative distances to potential close and far classes (beach, terrain/vegetation, foam and sky, respectively), excluding the smallest ones. So, compute boundaries and areas of each spots. (iii) Determine potential warning based on absolute low thresholds (to be validated at each site) and/or consecutive percentage differences between successive hours/days. Georeference information are useful for reporting useful data as in meters square rather than pixel, by means of standard method for georectification (Valentini, Saponieri, \& Damiani, 2017) at mean sea level.

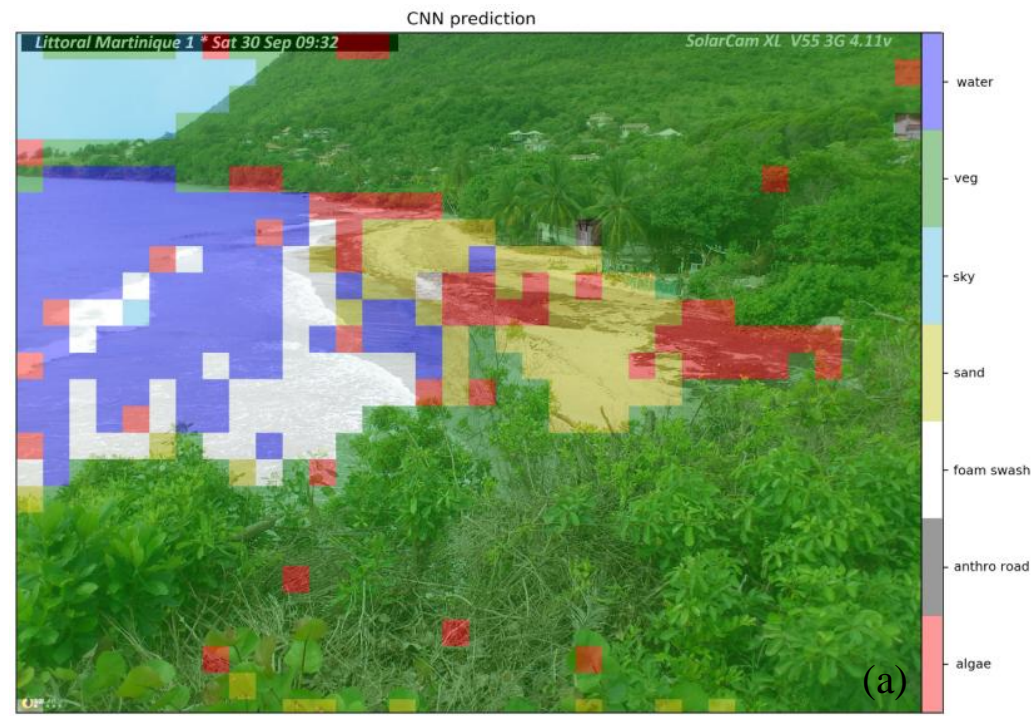



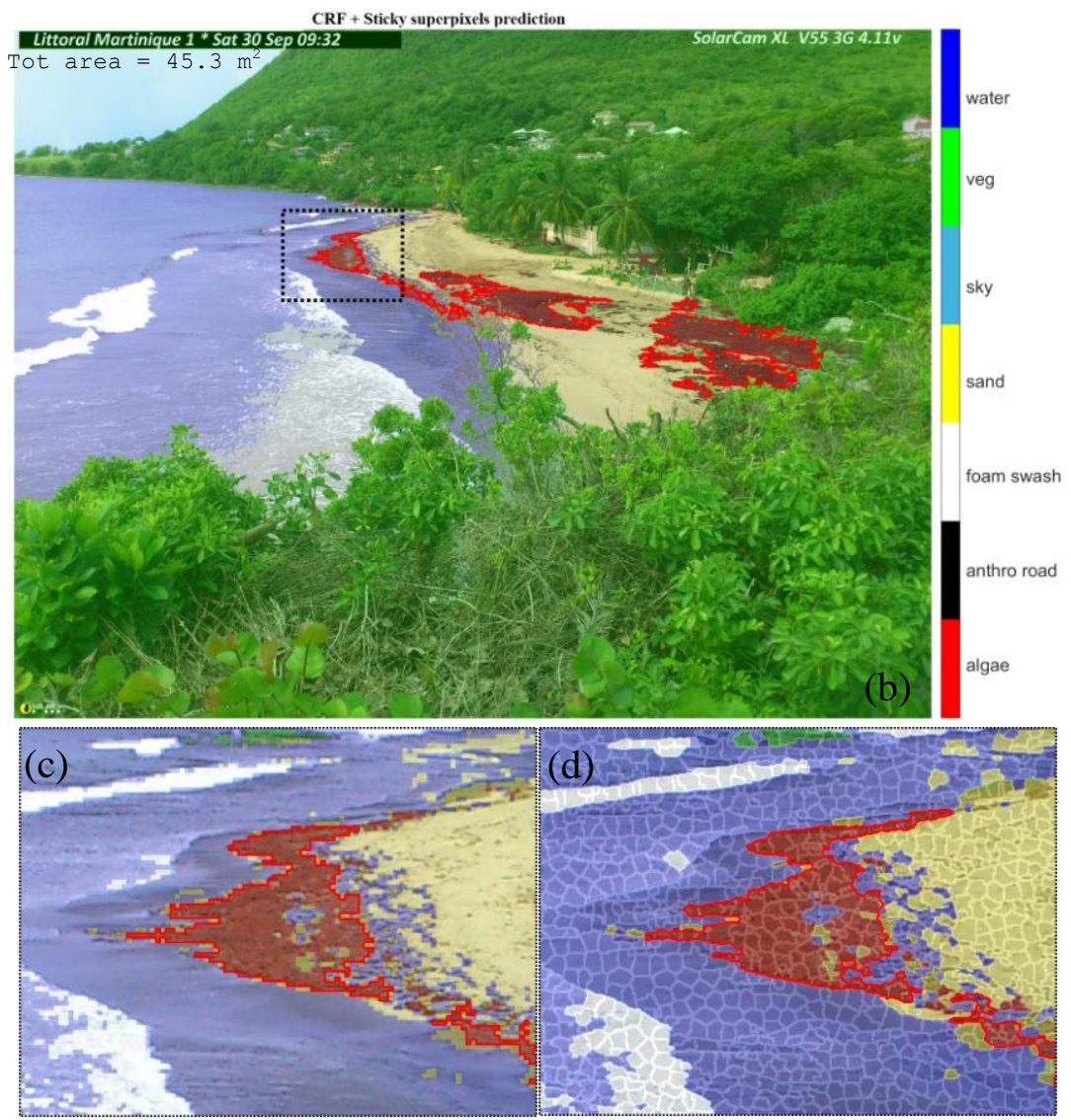

Fig. 5. Automatic Sargassum detection useful for the warning system under development for the Carribean coasts (Martinique and Guadaloupe). Chain example for Anse Cafard beach, Le Diamant, Martinique. (a). DCNN prediction on image patches, 96 pixels sized. The labels and colors are described at the right. (b). CRF + Sticky superpixel processes output with detected sargassum overlay. (FR). (c). Detail box from the lonely output of CRF process. (d). Same detail after applying the contours enhancement procedure by using the sticky superpixels.

The described warning system procedure is being tested at several sites in Martinique (FR), including three beaches and five coastal basins, for beached/bloom macroalgae spots detection. As an example, some qualitative results are presented in Fig. 5, which shows the spotted beached Sargassum at the beach of Anse Cafard, Le Diamant, Martinique. The processes are highlighted in the figures: from top to bottom, the DCNN (a) and the CRF + sticky edge superpixels predictions (b). A dashed rectangle box is overlay on the latter, in which the semantic contours detection is applied. Fig. $5 \mathrm{c}$ represents the segmentation as solely CRF output, the (d) is the coupled CRF + sticky 
superpixels. As partly illustrated, snapping to sticky superpixels further improves on boundary locations, as well as on noisy semantic segmentation detections in places where the superpixels are not present.

\section{Conclusion}

We described in this study the capabilities of smartphone-based low-cost video monitoring system, CSolarcam, aiming the investigation of coastal parameters. Results related to the description of the general dynamics of Posidonia, characterized by two general mechanisms of deposit. The relationship of nearshore algae dynamics with submerged morphological pattern such as sandbar(s) has been briefly presented. A new approach for semantic segmentation of coastal image has been highlighted in Sec. 3. The labelling, DCNN training and prediction routines have been presented. The drawbacks associated to the low spatial accuracy of DCNN segmentation has been alleviate with CRF detailing. Aiming at increasing contours precision for detection of fundamental boundaries (i.e. shoreline, algae spots, sand limits, etc.) the edges snapping procedure has been introduced. Results improve consistently, as outlined qualitatively in Sec. 4. The presented scheme represents an alternative methodological frame for coastal image analysis. Particularly, with its large range of applications: automation of tasks such as shoreline, surf area, macroalgae and shoreface detection and classification. This method has been implemented over a very simple, low-cost monitoring system and would be beneficial for a variety of scopes. Further research in hyperparameters optimization while training the model will assess a better classification of such morphological features particularly those smaller than an image patch (96 sq. pixel).

\section{Aknowledgements}

The video system used in this study were deployed as part of the Posidonia beach litter monitoring project funded by BRGM and DDTM 2B (Dir. Dep. Des Territoires et de la MEr de Haute-Corse), and the Sargassum monitoring network of Martinique funded by BRGM, ADEME (Ag. De L'environnement et de la maîtrise de l'Energie) and DEAL (Dir. Environnement, Aménagement et Logement) de Martinique.

\section{References}

Abadi, M., Barham, P., Chen, J., Chen, Z., Davis, A., Dean, J., ... Zheng, X. 
(2016). TensorFlow: A system for large-scale machine learning. Retrieved from http://arxiv.org/abs/1605.08695

Balouin, Y., Bélon, R., Merour, A., \& Riotte, C. (2014). Evolution of Corsican pocket beaches. Journal of Coastal Research, 70, 96-101.

https://doi.org/10.2112/SI70-017.1

Balouin, Y., Longueville, F., \& Colombet, Y. (2016). Video assessment of nearshore and beach evolution following the deployment of a submerged geotextile wave breaker. Journal of Coastal Research, Special Issue, 75(75), 617-621. https://doi.org/10.2112/SI75-124.1received

Bergh, M. Van den, Boix, X., Roig, G., \& Van Gool, L. (2013). SEEDS: Superpixels Extracted via Energy-Driven Sampling. Retrieved from http://arxiv.org/abs/1309.3848

Buscombe, D., \& Ritchie, A. (2018). Landscape Classification with Deep Neural Networks. Geosciences, 8(7), 244. https://doi.org/10.3390/geosciences 8070244

Chen, L.-C., Papandreou, G., Kokkinos, I., Murphy, K., \& Yuille, A. L. (2014). Semantic Image Segmentation with Deep Convolutional Nets and Fully Connected CRFs. Retrieved from http://arxiv.org/abs/1412.7062

Cuevas, E., Uribe-Martínez, A., \& Liceaga-Correa, M. de los Á. (2018). A satellite remote-sensing multi-index approach to discriminate pelagic Sargassum in the waters of the Yucatan Peninsula, Mexico. International Journal of Remote Sensing, 39(11), 3608-3627. https://doi.org/10.1080/01431161.2018.1447162

Dollar, P., \& Zitnick, C. L. (2015). Fast Edge Detection Using Structured Forests. IEEE Transactions on Pattern Analysis and Machine Intelligence, 37(8), 1558-1570. https://doi.org/10.1109/TPAMI.2014.2377715

Hariharan, B., Arbelaez, P., Bourdev, L., Maji, S., \& Malik, J. (2011). Semantic contours from inverse detectors. In 2011 International Conference on Computer Vision (pp. 991-998). IEEE. https://doi.org/10.1109/ICCV.2011.6126343

He, K., Zhang, X., Ren, S., \& Sun, J. (2015). Deep Residual Learning for Image Recognition. Retrieved from http://arxiv.org/abs/1512.03385 
Krizhevsky, A., Sutskever, I., \& Hinton, G. E. (2012). ImageNet Classification with Deep Convolutional Neural Networks. Retrieved from http://papers.nips.cc/paper/4824-imagenet-classification-with-deepconvolutional-neural-networks

Laigre, T. (2017). Utilisation de la photographie time-lapse pour l'étude de la dynamique des banquettes de posidonie et de leur impact sur l'évolution du trait de côte. BRGM.

Liu, B., \& Wen, Y. (n.d.). CNN and CRF for Semantic Image Segmentation. Pdfs.Semanticscholar.Org. Retrieved from https://pdfs.semanticscholar.org/6284/46b9873a7f8c87dd00ddf34a22d29c 95194d.pdf

Maninis, K.-K., Pont-Tuset, J., Arbeláez, P., \& Van Gool, L. (2017).

Convolutional Oriented Boundaries: From Image Segmentation to HighLevel Tasks. Retrieved from http://arxiv.org/abs/1701.04658

Sandler, M., Howard, A., Zhu, M., Zhmoginov, A., \& Chen, L.-C. (2018). MobileNetV2: Inverted Residuals and Linear Bottlenecks. Retrieved from http://arxiv.org/abs/1801.04381

Valentini, N., Damiani, L., Molfetta, M. G., \& Saponier, A. (2016). New coastal video-monitoring system achievement and development. In Proceedings of the Coastal Engineering Conference (Vol. 35).

Valentini, N., Saponieri, A., \& Damiani, L. (2017). A new video monitoring system in support of Coastal Zone Management at Apulia Region, Italy. Ocean and Coastal Management, 142.

https://doi.org/10.1016/j.ocecoaman.2017.03.032 